\title{
A Rare Uterine Mass-case Report
}

\author{
N. Hephzibah Kirubamani ${ }^{1 *}$ and Shruthi Kamal ${ }^{2}$ \\ 'Professor, Obstetrics \& Gyneacology, Saveetha Medical college-Saveetha University, Chennai. \\ 2Professor, Surgery, Saveetha Medical college-Saveetha University, Thandalam, \\ Chennai, Tamil Nadu, 602 105; shruthivenkat2002@yahoo.co.in
}

\begin{abstract}
Fibroids, also known as leiomyomas, are the most common benign uterine neoplasms. Although benign, they can undergo malignant transformation. Metastatic breast cancer is rarely identified in a uterine leiomyoma. Herein we are reporting a case of a 52-year postmenopausal patient who presented with rapidly increasing huge abdominal mass with a size that was almost reaching xipisternum. She underwent modified radicalmastectomy for infiltracting intraductalcarrcinoma two years ago and was tamoxifen since then. After investigation like abdominal and Pelvic USG,C.T abdomen suggested Leiomyosarcoma and subtotal hysterectomy was done due to technical difficulty. Pathological analysis revealed metasatic lobular breast carcinoma involving the uterine fundus and leiomyoma. This case is reported for its rarity, as Metastasis of extragenital neoplasms to the uterus is extremely rare and lobular breast cancer metastasizing to a leiomyoma in a patient using tamoxifen.
\end{abstract}

Keywords: Uterine Mass, Metatasizing to Leiomyoma, Lobular Cancer of Leiomyoma.

\section{Introduction}

Leiomyoma is the commonest benign uterine neoplasm. Sarcoma is the most common malignant change in fibroid. Uterine metastases from extragenital cancer are rarely reported. More frequently, the female genital tract is a direct extension from an adjacent pelvic organ. Breast cancer is the most common malignant disease in women, accounting for 192370 cases in 2009 in the United States [1]. However, breast cancer metastating to the female genital tract is rather uncommon; when this occurs, the ovaries are usually involved $[2,3]$. Similarly, Metastasis to the uterus has been identified in patients with a history of infiltrating lobular carcinoma of the breast $[3,6]$. Through this case and review of literature we want to high light clinical and histological aspects of this rare pathology.

\section{Case Report}

We report a case of 52-year-old postmenopausal women who was referred from department of general surgery

\footnotetext{
* Corresponding author:
}

N. Hephzibah Kirubamani (hepsi1002@yahoo.co.in) to gynaec outpatient department for a rapidly growing palpable mass abdomen up to xiphisternum. (Figure 1) The patient had no bowel disturbance and Micturation was normal. There were no pressure symptoms. The patient underwent Modified Radical Mastectomy for infiltrating ductal carcinoma of the right breast two years ago (Figure 1) and she was taking Tamoxifen since then and during her follow up abdominal USG revealed fibroid and she was advised hysterectomy about one and half years ago but the patient did not come for further follow-up. Physical examination revealed an obese woman with thyroid and other breast clinically normal. Vitals were stable. Per abdominal examination revealed a huge hard mass filling the entire abdomen arising from pelvic mass but no ascities. No organomegaly. Per vaginal examination revealed cervix hitched against pubic symphysis, Movement of cervix transmitted to mass in the abdomen. Speculum examination cervix normal. Routine laboratory investigations were normal, Abdominal and Pelvic USG (Figure 2 )shows large sized heterogenous mass arising from uterus with some changes 
near fundus suggestive of Leiomyoma with sarcomatous changes, both ovaries normal. No evidence of Ascities. Right side hydronephrosis.

C.T Abdomen and pelvis reveled heterogeneous mass arising from uterus, both ovaries normal, no ascities, few Ext iliac nodes were present, suggestive of Leiomyoma with sarcomatous changes. Hence Provisional clinical diagnosis of Leomyosarcoma was made.

Endometrial scrapping- nil, Cervix-normal. PapSmear report - Inflammatory. Ureteric stent was placed before exploratory Laparotomy and it revealed the presence of irregularly enlarged uterus about 34-36 weeks one more fibroid about $6 \mathrm{~cm}$ in the lower part of the uterus close to

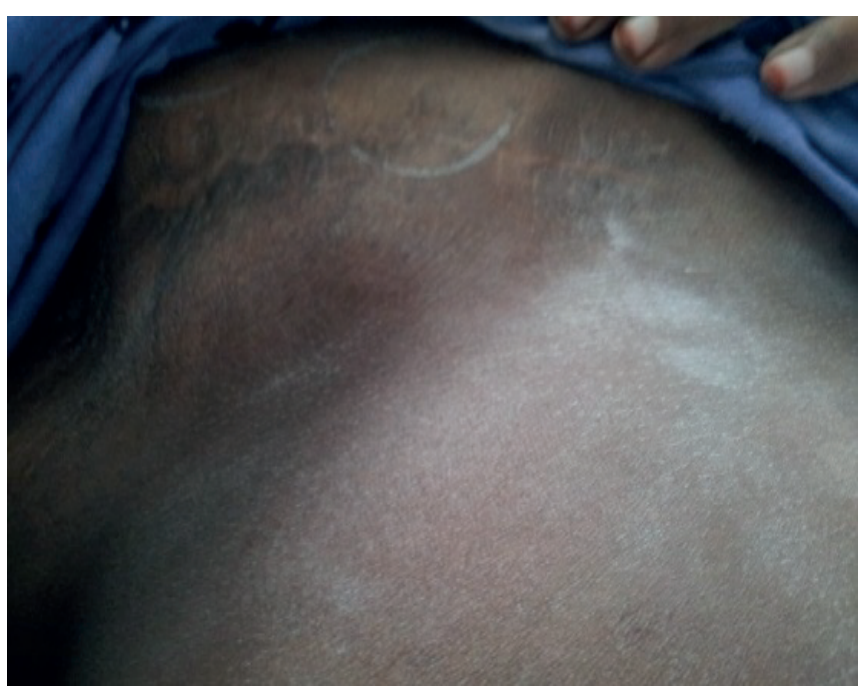

Figure 1. Huge abdominal mass with previous modified radical mastectomy scar.

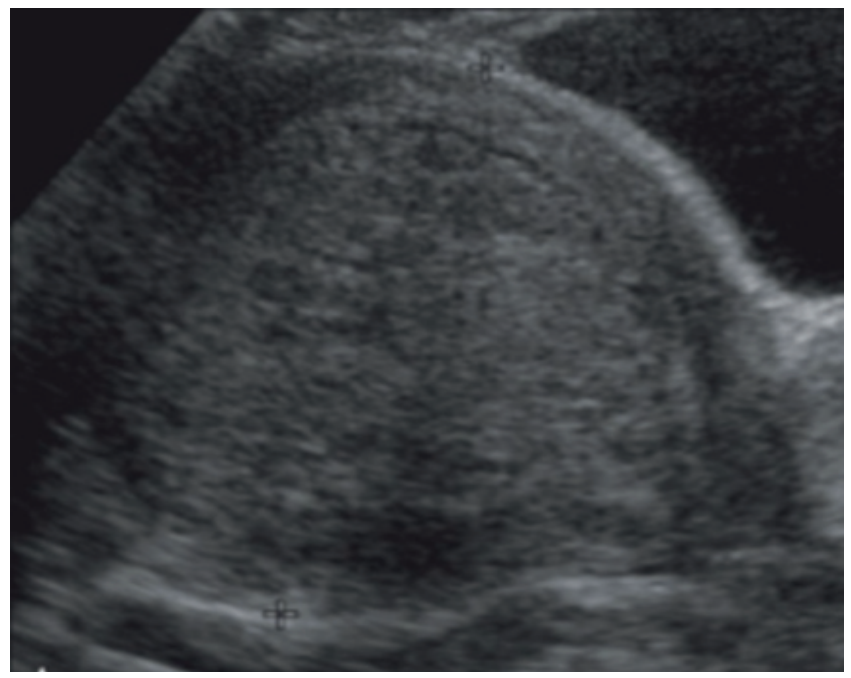

bladder, no Ascities (Figure 3). Both ovaries were normal and were laterally placed. Saline washing was sent for Cytological examination. Myomectomy followed by subtotal hysterectomy was done since there was another fibroid near bladder (Figure 4, 5 \& 6). As there was generalized ooze and patient general condition was not satisfactory, internal iliac artery ligation was carried out. Patient was given 3 units of blood on the table and also given with post-operative 3units of blood with FFP in 3 units. On 12th post-operative day, patient developed sub-acute intestinal obstruction due to adhesions and later had an abdominal wall dehiscence. She was taken up for relook laparotomy and bowel resection anastomosis, infracolic omentectomy was done and sent for HPE. The peritoneal and skin edges were trimmed and sutured. She had uneventful postoperative recovery and was discharged on $24^{\text {th }}$ day and sent for chemotherapy since pathologic analysis revealed metastatic lobular breast carcinoma involving the uterine fundus and a leiomyoma. (Figure 7).

\section{Discussion}

Metastasis of extragenital neoplasms to the uterus is extremely rare. For Lobular breast cancers, the chance to metastasize the uterus is more than the ductal carcinomas, but they metastasize as tiny nodules that can be missed with the standard diagnostic workup. Here we report a case of lobular breast cancer metastasizing to a leiomyoma in a patient using Tramoxifen.

Metastatic breast cancer occurs via lymphatic involvement, hematogeneous dissemination, and contiguous

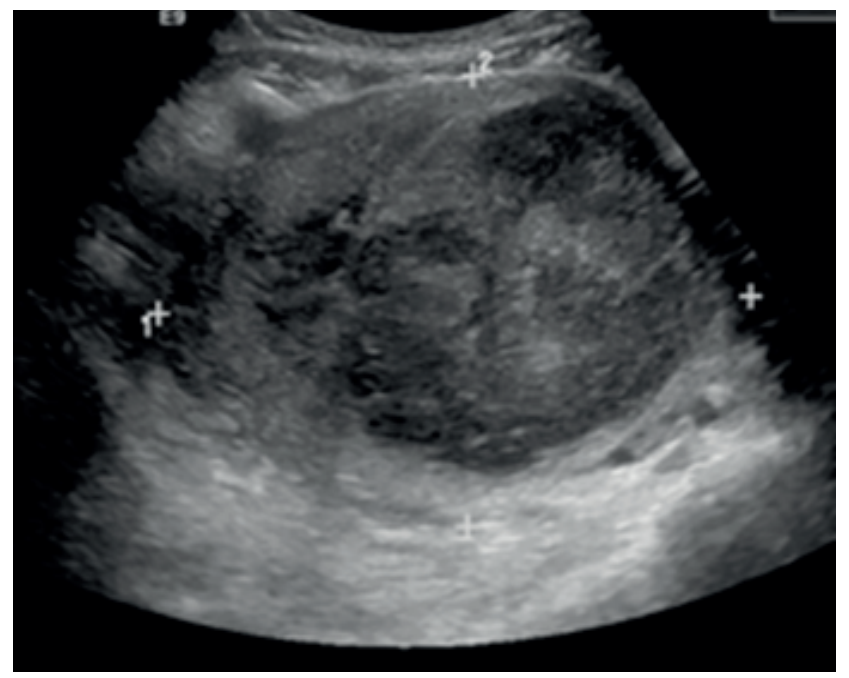

Figure 2. Abdominal USG shows huge fibroid changes near fibroid. 


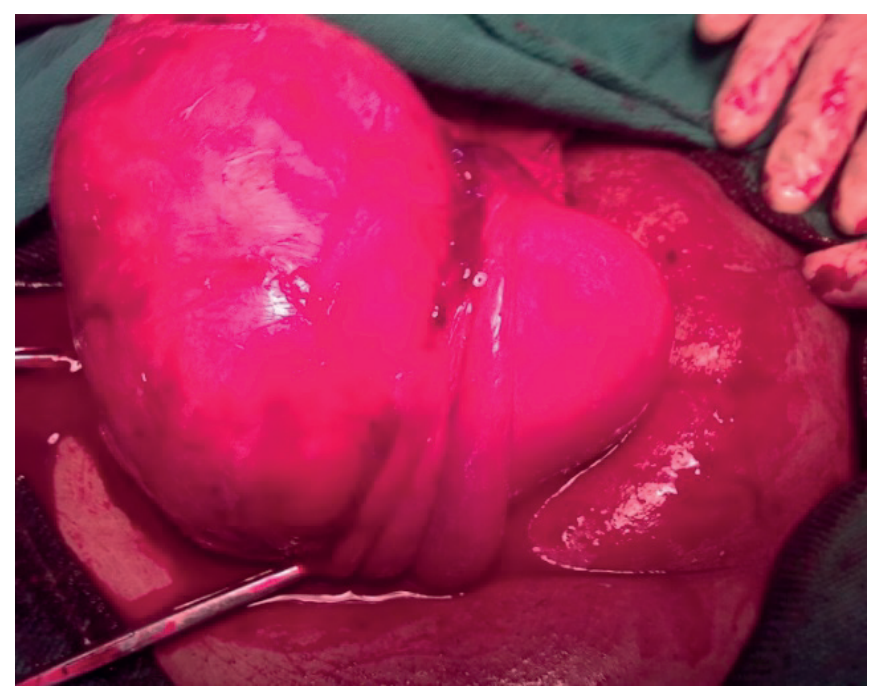

Figure 3. Peroperative findings.

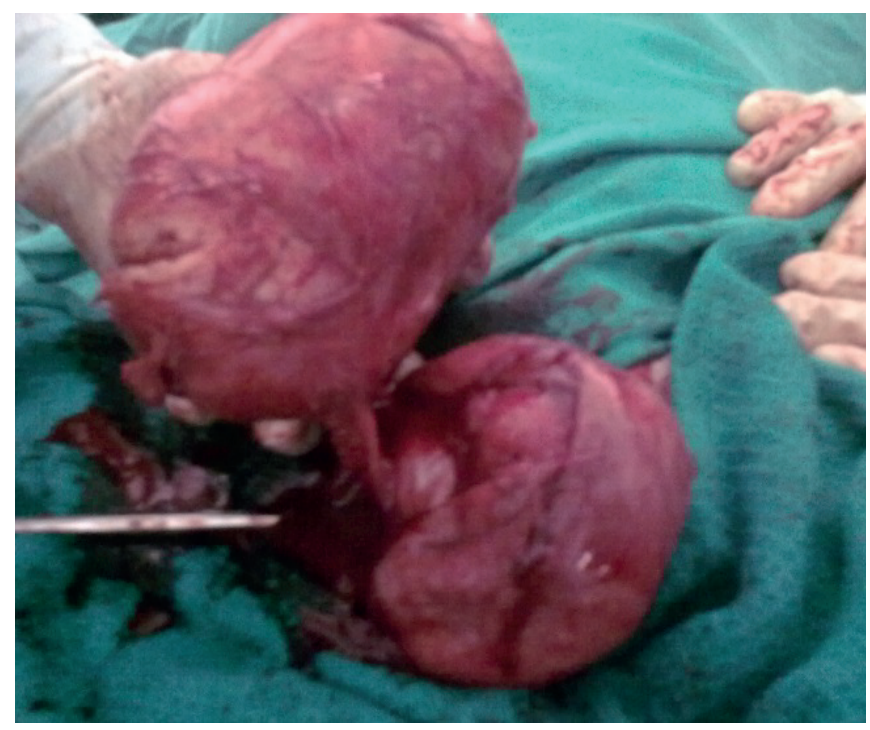

Figure 4. Myomectomy proceed with subtotal hysterectomy.

spread [5]. When the process is manifested, pulmonary, hepatic, and bone are the most common distal sites. If the gynecologic tract is involved, lobular carcinoma is more likely the source of the metastasis, rather than ductal carcinoma $[6,7]$.

One interesting aspect of our report is the metastatic breast cancer which has invaded the uterine myoma alone with no invasion of surrounding structures. There was no evidence of widespread disease and metastatic involvement but only within the uterus and Leiomyoma. Omentum specimen showed no evidence of malignancy: Ascitic fluid is negative for malignancy. Rest of the viscera is normal.

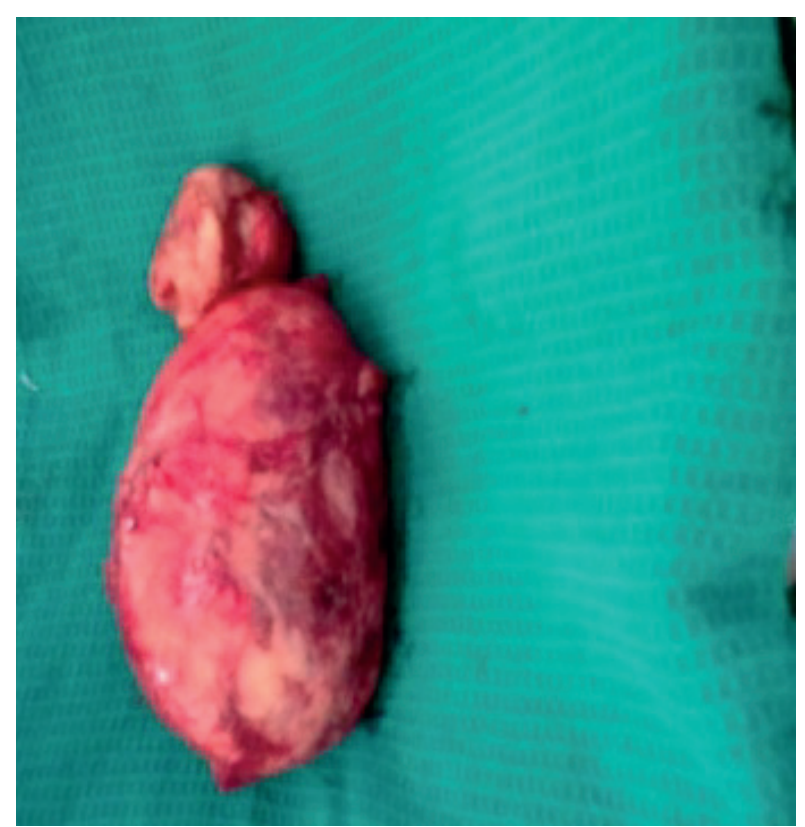

Figure 5. Myomectomy specimen.

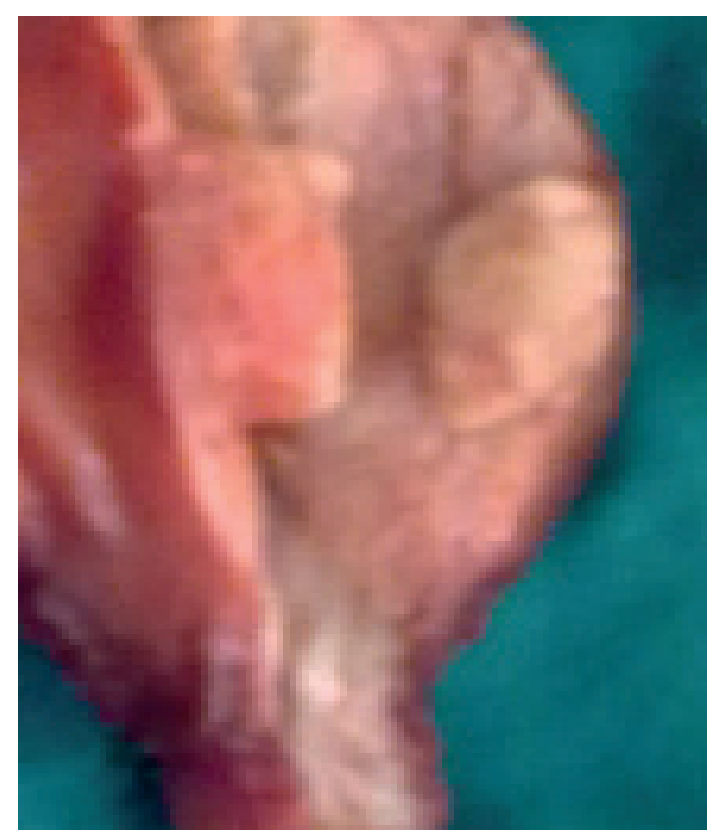

Figure 6. Subtotal hysterectomy specimen.

The other interesting fact we report is that infiltrating ductal breast carcinoma metastasizing on a uterine Leiomyoma, which is similar to earlier report [11]. Yasuhara et al. [11] described an advanced invasive ductal breast carcinoma in a patient who exhibited coexistent metastatic foci within a uterine leiomyosarcoma. Approximately, 22 cases of metastatic breast cancer that infiltrated a uterine Leiomyoma have been reported $[8,9,10]$. 

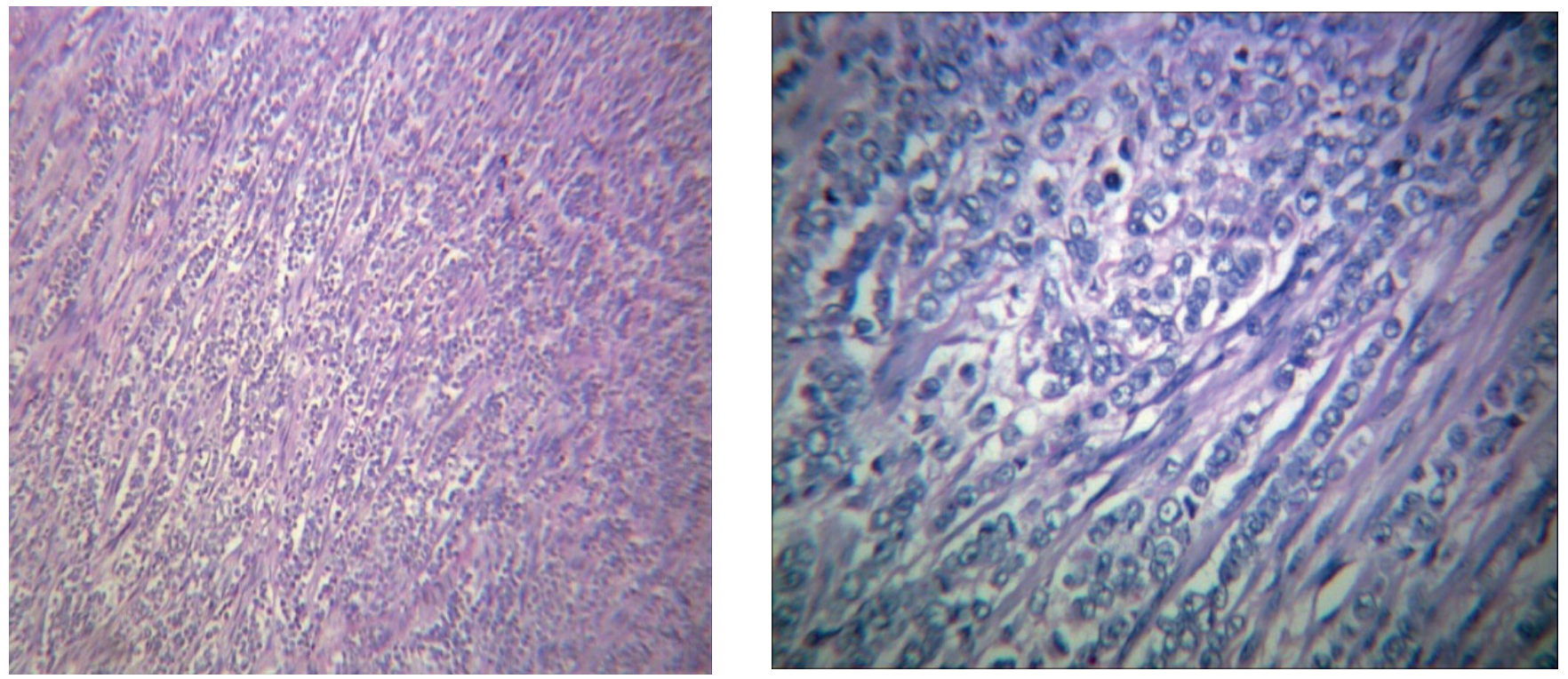

Figure 7. Histopathology examination shows Metastatic lobular breast carcinoma involving the uterine fundus and a leiomyoma (-cohesive cells individually dispersed or arranged in single-file linear patterns, 'Indian files).

However, when the leiomyosarcoma was initially diagnosed at imaging, the metastatic disease had yet to be identified. In another case study, Minelli et al. [8] reported a 37-year-old patient with a uterine wall myoma and hypermenorrhea. After surgical removal of the uterine myoma and pathologic review, a diagnosis of metastatic carcinoma of breast origin was made [4].

Histologic review of breast cancer subtypes also suggests that lobular carcinoma has a propensity for genital tract infiltration, predominantly in patients positive for hormone receptor $[12,13]$.

\section{Conclusion}

This entity can be confused clinically with a leiomyosarcoma. Growth of leiomyomas under aromatase inhibitors should be considered as a sign of metastases and surgery can be planned in selected cases.

\section{Acknowledgement}

The authors thank Chancellor, Pro-chancellor, Director \& Dean, of Saveetha Medical college for the encouragement and for giving permission to report the case. We would like to thank profusely Prof. Shruthi Kamal Prof of Surgery for the help and support to manage the case. We also thank sincerely Assistant surgeons Dr. Anupama, Dr. Padmabharathi and Anestheology Department for their earnest service.

\section{References}

1. American Cancer Society (2009). Cancer Facts \& Figures, Atlanta, GA.

2. Pauer H U, Viereck V et al. (2003). Uterine cervical metastasis of breast cancer: a rare complication that may be overlooked, Onkologie, vol 26(1), 58-60.

3. Scopa C D, Aletra C, et al. (2005). Metastases of breast carcinoma to the uterus: report of two cases, one harboring a primary endometrioid carcinoma, with review of the literature. Gynecologic Oncology, vol 96(2), 543-547.

4. Kumar N B, and Hart W R (1982). Metastases to the uterine corpus from extragenital cancers: a clinicopathologic study of 63 cases. Cancer, vol 50(10), 2163-2169.

5. Metser U, Haider M A et al. (2003). MR imaging findings and patterns of spread in secondary tumor involvement of the uterine body and cervix. American Journal of Roentgenology, vol 180(3), 765-769.

6. Borst M J, and Ingold J A (1993). Metastatic patterns of invasive lobular versus invasive ductal carcinoma of the breast. Surgery, vol 114(4), 637-641.

7. Lamovec J, and Bracko M (1991). Metastatic pattern of infiltrating lobular carcinoma of the breast: an autopsy study. Journal of Surgical Oncology, 48: 28-33.

8. Minelli L, Romagnolo C, et al. (1998). Uterine leiomyoma metastasis as a first sign of breast cancer, The Journal of the American Association of Gynecologic Laparoscopists, vol 5(2), 213-215.

9. Beattie G J, Duncan A J, et al. (1993). Breast carcinoma metastatic to uterine leiomyoma, Gynecologic Oncology, vol 51(2), 255-257. 
10. Sugiyama T, Toyoda N et al. (1995). Breast cancer metastatic to uterine leiomyoma: a case report, The Journal of Obstetrics and Gynaecology Research, vol 21(4), 349-355.

11. Yasuhara Y, Mikami Y et al. (2008). Metastatic breast carcinoma identified in a uterine leiomyosarcoma, Pathology International, vol 58(5), 317-321.
12. Kondo N I, Yoshida S et al. (2009). Metastasis of breast cancer to a uterine leiomyoma, Breast Cancer, vol 16(2), 157-161.

13. Piura B, Yanai-Inbar I et al. (1999). Abnormal uterine bleeding as a presenting sign of metastases to the uterine corpus, cervix and vagina in a breast cancer patient on tamoxifen therapy, European journal of obstetrics, gynecology, and reproductive biology, vol 83(1), 57-61. 